\title{
A10 IDENTIFICATION OF SPECIFIC CITRULLINATION SITES ON FIBRINOGEN IN RA
}

E Ossipova, ${ }^{1}$ V Malmström, ${ }^{1}$ A I Catrina, ${ }^{1}$ L Klareskog, ${ }^{1}$ R Zubarev, ${ }^{2}$ P-J Jakobsson ${ }^{1}$ Department of Medicine, Unit of Rheumatology, Karolinska University Hospital, Stockholm, Sweden; ${ }^{2}$ Department of medical biochemistry and biophysics; Karolinska Institutet, Stockholm, Sweden

10.1136/ard.2010.129577j

Background Anti-citrullinated protein/peptide antibodies are highly specific serological markers for rheumatoid arthritis. Citrullination is a post-translational modification of charged arginine residues to neutral citrulline by peptidyl arginine deiminases. Induced expression of citrullinated proteins are frequently detected in various inflammatory states including arthritis by anti-citrulline antibodies; however, direct identification and quantification of citrullinated residues in arthritic samples has not been successfully performed in the past.

Objectives The aim of this project was to identify by highresolution mass spectrometry citrullinated proteins and within them, to identify the citrullination sites and quantify their occupancies in synovial tissue from rheumatoid arthritis patients. 
Methods The authors chose to study citrullination of fibrinogen, a candidate auto antigen in arthritis, as it is commonly targeted by anti-citrullinated protein/peptide antibodies. Synovial tissues from arthritis patients were sequentially extracted to enrich fibrinogen. The extracts were pooled and highly abundant proteins removed by immunodepletion. The remaining protein sample was analysed by one dimensional electrophoresis and liquid chromatography coupled mass spectrometry.

Results A peptide with the sequence corresponding to amino acids 559-575 of fibrinogen $\alpha$ chain was identified to be citrullinated with an occupancy rate of $1.4-2.5 \%$. Furthermore, citrullination of the peptide corresponding to amino acids 52-77 of the fibrinogen $\beta$ chain was identified in one patient sample with an occupancy rate of $1.2 \%$ (Hermansson et al, 2010, PROTEOMICS - Clinical Applications (2010) DOI: 10.1002/ prca.200900088). The identifications were made using in vitro citrullinated fibrinogen and accurate mass and retention time analysis. The authors are now investigating the occurrence of autoantibodies recognising these citrulline containing peptides in RA and preliminary results will be presented.

Conclusion Detailed studies on which molecules are citrullinated in arthritis and other inflammatory states can provide information about their role in immune regulation and serve as novel biomarkers and potentially even as therapeutic targets. 